\begin{tabular}{|c|l|}
\hline Title & N-Linked neutral oligosaccharides in the stratum corneum of normal and ichthyotic skin \\
\hline Author(s) & $\begin{array}{l}\text { Ito, Hiroko; A kiyama, Masashi; Nakagawa, Hiroaki; Uematsu, Rie; Deguchi, Kisaburo; McMillan, James R.; } \\
\text { Nishimura, Shin-Ichiro; Shimizu, Hiroshi }\end{array}$ \\
\hline Citation & $\begin{array}{l}\text { Archives of Dermatological Research, 298(8), 403-407 } \\
\text { https://doi.org/40.1007/300403-006-0702-0 }\end{array}$ \\
\hline Issue Date & 2007-01 \\
\hline Doc URL & http://hdl.handle.net/2115/17126 \\
\hline Rights & The original publication is available at www.springerlink.com \\
\hline Type & article (author version) \\
\hline File Information & ADR298-8.pdf \\
\hline
\end{tabular}

Instructions for use 


\section{Archives of Dermatological Research}

Manuscript No. ADR-06-0063 Revised Version

\section{Short Communication}

\section{$\mathrm{N}$-linked neutral oligosaccharides in the stratum corneum of normal and ichthyotic skin}

Hiroko Ito ${ }^{1}$, Masashi Akiyama ${ }^{1}$, Hiroaki Nakagawa ${ }^{2}$, Rie Uematsu ${ }^{2,3}$, Kisaburo Deguchi ${ }^{2}$, James R. McMillan ${ }^{1,4}$, Shin-Ichiro Nishimura ${ }^{2}$ and Hiroshi Shimizu $^{1}$

${ }^{1}$ Department of Dermatology, Hokkaido University Graduate School of Medicine, Sapporo, Japan

${ }^{2}$ Division of Biological Sciences, Frontier Research Center for Post-Genomic Science and Technology, Hokkaido University Graduate School of Science, Sapporo, Japan

${ }^{3}$ Research Laboratory, Kanebo COSMETICS INC., Odawara, Japan ${ }^{4}$ Creative Research Initiative Sousei, Hokkaido University, Sapporo, Japan

Word count (text only): 1720 words

Grant support: This work was partly supported by a grant for the National Project on "Functional Glycoconjugate Research Aimed at Developing New Industry” from the Ministry of Education, Science, Sport and Culture of Japan.

\section{Correspondence and reprint requests to:}

Masashi Akiyama, MD, PhD

Department of Dermatology

Hokkaido University Graduate School of Medicine

North 15 West 7, Sapporo 060-8638, Japan

Telephone: +81-11-716-1161, ext. 5962 Fax: +81-11-706-7820

e-mail <akiyama@med.hokudai.ac.jp> 


\begin{abstract}
$\mathrm{N}$-glycan oligosaccharides are thought to play multiple, important roles in a variety of biological events. However, $N$-glycan profiles in the stratum corneum of human skin have not yet been studied in detail. To clarify the $\mathrm{N}$-glycan profiles in the stratum corneum of normal and ichthyotic epidermis, $N$-glycan profiles were studied by high-performance liquid chromatography (HPLC) using normal human epidermal samples and scales from hyperkeratotic skin of ichthyosis patients. Chromatograms of patient scale samples showed unique alterations in three peaks eluted at 15.8, 18.8 and $26.9 \mathrm{~min}$. The $N$-glycan profiles were significantly altered in ichthyotic hyperkeratotic skin compared with normal non-hyperkeratotic controls. These findings indicate reduction of $\mathrm{N}$-acetylglucosaminyltransferase II and fucosyltransferase 8 activities. Alteration of $N$-glycan structures in hyperkeratotic skin suggests biological role for $N$-glycans in keratinization.
\end{abstract}

Keywords: high-performance liquid chromatography, hyperkeratosis, neutral oligosaccharide, $N$-glycan, oligosaccharide. 
Oligosaccharides are known to play important roles in a variety of biological events including cell-cell interactions and undergo modifications during cell differentiation [6]. $N$-glycan oligosaccharides, which bind to asparagine, are a frequent post-translational modification of proteins. $\mathrm{N}$-glycan oligosaccharides exhibit diversity created by sugar linkages and components including: mannose, galactose, $N$-acetylglucosamine, $\mathrm{N}$-acetylneuraminic acid. $\mathrm{N}$-glycan oligosaccharides modify specific protein properties, especially glycoprotein localization, secretion and degradation rates of proteins. Alterations in $\mathrm{N}$-glycan composition have been reported in many human diseases [6, 12]. Oligosaccharide analyses using oligosaccharide-specific lectins and antibodies have suggested the biological importance of oligosaccharides in the epidermis [3, 5, 7, 19-21, 25, 28]. However, oligosaccharide-specific lectins and antibodies recognize only two or three sugar residues at most and little information has been obtained from studies with lectins or antibodies concerning the entire range of glycan structures or the $\mathrm{N}$-glycan and $O$-glycan composition on glycoproteins and glycolipids. The cellular biosynthesis of $N$-glycan is highly regulated and glycan structures significantly affect protein properties $[6,15]$. Alteration of $N$-glycan profiles in diseases have been reported in many human disorders, and these have been used for disease diagnosis and for elucidation of disease pathomechanisms $[4,8]$. Focusing on glycoproteins $N$-glycans, it was demonstrated that the mouse epidermis had abundant high-mannose oligosaccharides [24]. In addition, the oligosaccharides binding proteins were also determined [24]. However, there are no reports of studies on $N$-glycans in human epidermis. In the present study, we studied $N$-glycan profiles in normal human epidermis and the stratum corneum of ichthyotic, 
hyperkeratotic skin. $N$-glycans were specifically released by $N$-glycosidase F and were labeled by pyridylamination with a fluorescence tag. Oligosaccharide profiles were analyzed using high-performance liquid chromatography (HPLC) [10, 22].

Skin samples of normal, non-hyperkeratotic epidermis were collected from control non-palmoplantar skin of three patients with benign skin tumors at resection operations. Skin scale samples were collected from hyperkeratotic skin on the legs of five ichthyosis patients, three non-bullous congenital ichthyosiform erythroderma patients and two lamellar ichthyosis patients. The medical ethical committee at Hokkaido University approved all described studies. All the participants or parents of the participants gave their written informed consent.

Skin scales were collected by gently scraping patients' legs with a dull scalpel and stored at $-70{ }^{\circ} \mathrm{C}$. Normal epidermal samples were obtained at resection operations of benign subcutaneous tumors after obtaining fully informed consent from the patients. Both samples from ichthyosis patients and normal healthy controls were stored at $-70^{\circ} \mathrm{C}$.

Normal skin samples were heated to $60^{\circ} \mathrm{C}$ for $1 \mathrm{~min}$ in phosphate buffered saline (PBS) and then cooled on ice. The epidermis was stripped off with a scalpel and was used for further analysis. The epidermis of normal skin samples was washed 3 times in PBS. Both scales and the separated epidermis were denatured at $90{ }^{\circ} \mathrm{C}$ for $10 \mathrm{~min}$ in $100 \mathrm{mM}$ $\mathrm{NH}_{4} \mathrm{HCO}_{3}$ and then finely shredded with scissors. 
To degrease the samples, each sample was put into a mixture of chloroform and methanol 2:1 (v/v) $600 \mu \mathrm{l}$, mixed well, and centrifuged at $3500 \mathrm{rpm}, 4^{\circ} \mathrm{C}$ for $10 \mathrm{~min}$. Then, the lower layer was removed. This procedure was repeated twice to remove lipids. Organic solvents in the upper layer were vaporized with nitrogen flow at $80^{\circ} \mathrm{C}$ and the aqueous solution dried in a centrifugal evaporator.

For trypsin and chymotrypsin digestion, dried samples were suspended in $5 \mathrm{mg}$ per $100 \mu \mathrm{l}$ of $45 \mathrm{mM}$ Tris-HCl buffer $\mathrm{pH} 8$ containing $10 \mathrm{mM}$ $\mathrm{CaCl}_{2}$, and $0.1 \mathrm{mg}$ trypsin and $0.1 \mathrm{mg}$ chymotrypsin (Sigma-Aldrich Japan, Tokyo, Japan) were added in a $1.5 \mathrm{ml}$ microcentrifuge tube (Eppendorf type). The $\mathrm{pH}$ of the solutions was confirmed to be between 7 and 8 by $\mathrm{pH}$ test paper. Toluene was added to the surface of the sample solution and kept at $37^{\circ} \mathrm{C}$ overnight in an incubator.

For $N$-glycosidase F digestion, trypsin and chymotrypsin were deactivated by heating sample tubes at $90^{\circ} \mathrm{C}$ for $10 \mathrm{~min}$ in a heat block. $N$-glycosidase F (Roche Diagnostics, Tokyo, Japan) was added, 10 units for each $5 \mathrm{mg}$ sample. The $\mathrm{pH}$ of the sample solutions was again confirmed and toluene was added. The samples were kept at $37^{\circ} \mathrm{C}$ overnight.

$0.1 \mathrm{mg}$ of pronase (Calbiochem, San Diego, U.S.A.) was added to each $5 \mathrm{mg}$ of the sample for pronase digestion. $\mathrm{pH}$ of the sample solutions was checked again and toluene was added. The samples were incubated at $37^{\circ} \mathrm{C}$ overnight. After incubation, pronase was deactivated at $90{ }^{\circ} \mathrm{C}$ for $10 \mathrm{~min}$.

The oligosaccharide fraction was purified by gel-filtration on Bio-gel 
P-4 (Bio-Rad Laboratories, Hercules, California, U.S.A.) column (1.0 x 38 $\mathrm{cm}$ ) using water as an eluate, and lyophilized completely. Carbohydrates were reductively aminated with 2-aminopyridine and sodium cyanoborohydride [10, 29]. Pyridylaminated (PA) oligosaccharides were separated from excess reagents by another gel-filtration column step using Sephadex G-15 (GE Healthcare Bioscience Corp., Piscataway, NJ, U.S.A.) and $10 \mathrm{mM} \mathrm{NH} \mathrm{HCO}_{3}$ and lyophilized again.

Each PA-oligosaccharide was dissolved in $100 \mu \mathrm{l}$ of $0.01 \mathrm{~N} \mathrm{HCl}$ and $\mathrm{pH}$ was adjusted to 2 with $0.1 \mathrm{~N} \mathrm{HCl}$ checking by test paper.

PA-oligosaccharide solutions were heated at $90{ }^{\circ} \mathrm{C}$ for 60 min to remove sialic acids and neutralized with $100 \mu \mathrm{l}$ of $1 \mathrm{M} \mathrm{NH}_{4} \mathrm{HCO}_{3}$.

Each PA-oligosaccharide solution was purified by HPLC on an amide-silica column (TSKgel Amide-80 4.6 x 250 mm, Tosoh Corporation, Tokyo, Japan) using two solvents, A and B at a flow rate of $1.0 \mathrm{ml} / \mathrm{min}$ at $40{ }^{\circ} \mathrm{C}$. Solvent A comprised $65 \%(\mathrm{v} / \mathrm{v})$ of acetonitrile and $35 \%$ of $0.5 \mathrm{M}$ acetic acid-triethylamine buffer pH 7.3 and solvent B was $35 \%$ and $65 \%$, respectively. The column was equilibrated only with solvent A and 7 min after injection of each sample, the flow was changed to solvent B only. The $\mathrm{N}$-glycan fraction that eluted between 8 to 12 min was collected and centrifugally evaporated.

Each oligosaccharide mixture was dissolved in water and analyzed by HPLC using the previously described procedure [22]. PA-oligosaccharide mixture was applied on an ODS column (HRC-ODS 6 x 150 mm, Shimadzu Corporation, Kyoto, Japan). The column was equilibrated with 
$0.1 \%$ (v/v) 1-butanol in $10 \mathrm{mM}$ sodium-phosphate buffer $\mathrm{pH} 3.8$ and concentration of 1-butanol was linearly increased to $0.25 \%$ in $60 \mathrm{~min}$. Flow rate was $1.0 \mathrm{ml} / \mathrm{min}$ and column was controlled at $55^{\circ} \mathrm{C}$. PA-glycans were detected by fluorescence, excitation at $320 \mathrm{~nm}$ and emission at 400 $\mathrm{nm}$.

$\mathrm{N}$-glycan profiles from the patients and normal control epidermis are shown in Fig. 1 as chromatograms on an ODS column. $N$-glycan eluted after 5.0 min and few contamination peaks were detected in this region. High-mannose type $N$-glycans eluted from $5 \mathrm{~min}$ to $10 \mathrm{~min}$ and complex-type and hybrid-type $\mathrm{N}$-glycans appeared at $10 \mathrm{~min}$ or later in this analysis. Three peaks, a, b and c eluted at 15.8, 18.8 and $26.9 \mathrm{~min}$ respectively and these profiles were significantly altered in ichthyotic skin. The rates of these peaks are shown in Figure 2. The peak c was significantly decreased and the peak b was increased in the ichthyotic skin compared with normal controls. Peak a was also larger in the patients, but the statistical change in this level was not significant. Using two dimensional mapping [22], these peaks a, b and c were suggested to correspond to major serum $N$-glycans, Gal $\beta 1,4$ GlcNAc $\beta 1,2 M a n \alpha 1,3(G a l \beta 1,4 G l c N A c \beta 1,2 M a n \alpha 1,6) M a n \beta 1,4 G l c$ NAc $\beta 1,4 G l c N A c$,

Gal $\beta 1,4 G l c N A c \beta 1,2$ Man $\alpha 1,3($ Man $\alpha 1,6) M a n \beta 1,4 G l c N A c \beta 1,4(F u c \alpha 1,6) G l$ cNAc and

Gal $\beta 1$,4GlcNAc $\beta 1,2$ Man $\alpha 1,3(G a l \beta 1,4 G l c N A c \beta 1,2 M a n \alpha 1,6) M a n \beta 1,4 G l c$ NAc $\beta 1,4($ Fuc $\alpha 1,6)$ GlcNAc respectively $[17,22]$.

Changes in $\mathrm{N}$-glycan oligosaccharides have mainly been reported in 
carcinomas in the course of progression or during metastatic change [13, 14, 23]. In the epidermis, glycoconjugate profile changes were studied by lectin histochemistry in a variety of skin diseases $[1,2,9]$. In several autoimmune skin diseases, autoantibody targets have been shown to be $N$-linked oligosaccharides [18]. However, to our knowledge, this is the first study that reported $N$-linked oligosaccharide profiles in normal and hyperkeratotic epidermis.

Glycosylation is the major posttranslational modification of many proteins. $N$-glycan precursor, Glc3Man9GlcNAc2, is introduced to asparagine residues of Asn-X-Ser/Thr sequence while polypeptide synthesis occurs in the endoplasmic reticulum. Glc residues are removed while protein holding occurs. High-mannose type $N$-glycans work as a tag in the transfer of a protein folded correctly to Golgi apparatus or misfolded one to degradation system [11]. In the Golgi apparatus, several enzymes modify $N$-glycan oligosaccharides from the high-mannose type into a more complex type by trimming and repetitive addition of sugar residues [15]. Biosynthesis of $N$-glycans is highly conserved and these oligosaccharide residues on proteins have important roles in promoting protein regulation, folding, quality control, sorting, and transport [6].

The $N$-glycan alterations seen in ichthyotic skin failed to suggest novel oligosaccharide structures, although the rates of known oligosaccharide structures were changed. Peak a is the most common $N$-glycan in human serum. Peak $\mathrm{b}$ is formed due to insufficient activity of $N$-acetylglucosaminyltransferase II (GnT-II) and peak c is produced from peak a $N$-glycan by fucosyltransferase 8 (Fut8) activity [6]. GnT-II 
knock-out mice were reported to die from serious defects of multiple organs in early postnatal development, mainly from abnormalities in the gastrointestinal tracts, hematopoietic systems and bones, although skin involvements were not apparent [27]. Fut8 null mice were also lethal in early postnatal days mainly from emphysema-like changes in the lung [26]. The Fut8 null mice were known to overexpress matrix metaloproteinases and to show function loss of TGF- $\beta$ receptor. TGF- $\beta$ mediates a variety of signaling pathways [16] and dysfunction of TGF- $\beta$ receptor may be associated with skin symptoms of ichthyosis in the present study. On the other hand, there is a possibility that disturbed differentiation of epidermal keratinocytes resulted in the altered $N$-glycan profiles in the ichthyotic lesions. In any case, the relationship between $N$-glycan alteration and hyperkeratosis is an interesting finding that may help to clarify the pathomechanism(s) of hyperkeratosis.

Epidermal proteins which comprise high levels of mannose type oligosaccharides were analyzed in mouse [24]. These high-mannose type oligosaccharides are located in lysosomes, lamellar granules and cell-cell connection desmosome sites. Desmocollins and desmogleins are also assigned as carriers of high-mannose type oligosaccharides, and it was suggested that high-mannose type oligosaccharides control desquamation [24]. However, in the present samples of hyperkeratotic skin, the profile of the high-mannose type oligosaccharides was not changed. These results indicated that $N$-glycans bound to cell surface proteins were more seriously affected than those in the cytoplasm and desmosomes. Further studies into $\mathrm{N}$-glycan structures and the respective proteins carrying the $\mathrm{N}$-glycans in hyperkeratotic skin will provide further clues to determine the exact 
mechanisms leading to hyperkeratosis in human skin.

\section{Acknowledgements}

Authors thank Ms. Mayuko Nagasaki for her excellent technical assistance.

This work was partly supported by a grant for the National Project on

"Functional Glycoconjugate Research Aimed at Developing New

Industry” from the Ministry of Education, Science, Sport and Culture of Japan. 


\section{References}

1. Akiyama M, Hayakawa K, Watanabe Y, Nishikawa T (1990)

Lectin-binding sites in clear cell acanthoma. J Cutan Pathol 17: 197-201

2. Akiyama M, Sugiura M, Shimizu H, Nishikawa T (1991) No significant change of glycoconjugates exists in the epidermis of familial benign chronic pemphigus. Arch Dermatol Res 283: 537-539

3. Bell CM, Skerrow CJ (19899 Factors affecting the binding of lectins to normal human skin. Br J Dermatol 111: 517-526

4. Butler M, Quelhas D, Critchley AJ, Carchon H, Hebestreit HF, Hibbert RG, Vilarinho L, Teles E, Matthijs G, Schollen E, Argibay P, Harvey DJ, Dwek RA, Jaeken J, Rudd PM (2003) Detailed glycan analysis of serum glycoproteins of patients with congenital disorders of glycosylation indicates the specific defective glycan processing step and provides an insight into pathogenesis. Glycobiology 13: 601-622

5. Dabelsteen E, Buschard K, Hakomori S-I, Young WW (1989) Pattern of distribution of blood group antigens on human epidermal cells during maturation. J Invest Dermatol 82: 13-17

6. Dwek RA (1996) Glycobiology: Toward understanding the function of sugars. Chem Rev 96: 683-720

7. Elias PM, Chung J-C, Orozco-Topete R, Nemanic MK (1983) 
Membrane glycoconjugate visualization and biosynthesis in normal and retinoid-treated epidermis. J Invest Dermatol 81: 81s-85s

8. Fukuda MN. Dell A, Scartezzini P (1987) Primary defect of congenital dyserythropoietic anemia type II. J Biol Chem 262: 7195-7206

9. Gheri G, Russo G, Cappugi P, Sgambati E, Bryk SG (1999) The oligosaccharidic component of the glycoconjugates in lichen planus, granuloma annulare, seborrheic keratosis and plamoplantar keratoderma: lectin histochemical study. Histol Histopathol 14: 697-704

10. Hase S, Ikenaka T, Matsushima Y (1978) Structure analyses of oligosaccharides by tagging of the reducing end sugars with a fluorescent compound. Biochem Biophys Res Commun 85: 257-263

11. Helenius A, Aebi M (2001) Intracellular functions of N-linked glycans. Science 291: 2364-2369

12. Helenius A, Aebi M (2004) Role of N-linked glycans in the endoplasmic reticulum, Annu Rev Biochem 73: 1019-1049

13. Ishimura H, Takahashi T, Nakagawa H, Nishimura S-I, Hagisawa S, Arai Y, Horikawa Y, Habuchi T, Miyoshi E, Ohyama C (2006) $N$-acetylglucosaminyltransferase-V (GnT-V) and $\beta$ 1,6 branching $N$-linked oligosaccharides are associated with good prognosis of the patients with bladder cancer. Clin Cancer Res 102: 15791-15796 
14. Jorgensen T, Berner A, Kaalhus O, Tveter KJ, Danielsen HE, Bryne M (1995) Up-regulation of the oligosaccharide sialyl LewisX: a new prognostic parameter in metastatic prostate cancer. Cancer Res 55: 1817-1819

15. Kornfeld R, Kornfeld S (1985) Assembly of asparagine-linked oligosaccharides. Ann Rev Biochem 54: 631-664

16. Massagué J, Blain SW, Lo RS (2000) TGF $\beta$ signaling in growth control, cancer, and heritable disorders, Cell 103: 295-309

17. Nakagawa H, Kawamura Y, Kato K, Shimada I, Arata Y, Takahashi N (1995) Identification of neutral and sialyl N-linked oligosaccharide structures from human serum glycoproteins using three kinds of high-performance liquid chromatography. Anal Biochem 226: 130-138

18. Shimanovich I, Hirako Y, Sitaru C, Hashimoto T, Brocker EB, Butt E, Zillikens D (2003) The autoantigen of anti-p200 pemphigoid is an acidic noncollagenous $\mathrm{N}$-linked glycoprotein of the cutaneous basement membrane. J Invest Dermatol 121: 1402-1408

19. Skerrow CJ, Clelland DG, Skerrow D (1989) Changes to desmosomal antigens and lectin-binding sites during differentiation in normal human epidermis: a quantitative ultrastructural study. J Cell Sci 92: 667-677 
20. Srikrishna G, Varki NM, Newell PC, Varki A, Freeze HH (1997) An IgG monoclonal antibody against Dictystelium discoideum glycoproteins specifically recognizes Fuc $\alpha 1$,6GlcNAc $\beta$ in the core of N-linked glycans. J Biol Chem 272: 25743-25752

21. Symington FW, Holmes EH, Symington BE (1992) Human epidermal keratinocyte expression of sialyl-Lewis X. J Invest Dermatol 99: 601-607

22. Tomiya N, Awaya J, Kurono M, Endo S, Arata Y, Takahashi N (1988) Analyses of N-linked oligosaccharides using a two-dimensional mapping technique. Anal Biochem 1715: 73-90

23. Tsuchiya N, Yamanaka R, Yajima N, Homma J, Sano M, Komata T, Ikeda T, Fujimoto I, Takahashi H, Tanaka R, Ikenaka K (2005) Isolation and characterization of an N-linked oligosaccharide that is increased in glioblastoma tissue and cell lines. Int J Oncol 27: 1231-1239

24. Uematsu R, Furukawa J-I, Nakagawa H, Shinohara Y, Deguchi K, Monde K, Nishimura S-I (2005) High throughput quantitative glycomics and glycoform-focused proteomics of murine dermis and epidermis. Mol Cell Proteomics 4: 1977-1989

25. Virtanen I, Kariniemi A-L, Holthöfer H, Lehto V-P (1986) Fluorochrome-coupled lectins reveal distinct cellular domains in human epidermis. J Histochem Cytochem 34: 307-315 
26. Wang X, Inoue S, Gu J, Miyoshi E, Noda K, Li W, Mizuno-Horikawa Y, Nakano M, Asahi M, Takahashi M, Uozumi N, Ihara S, Lee SH, Ikeda Y, Yamaguchi Y, Aze Y, Tomiyama Y, Fujii J, Suzuki K, Kondo A, Shapiro SD, Lopez-Otin C, Kuwaki T, Okabe M, Honke K, Taniguchi N (2005) Proc Natl Acad Soc USA 102: 15791-15796

27. Wang Y, Tan J, Sutton-Smith M, Ditto D, Panico M, Campbell RM, Varki NM, Long JM, Jaeken J, Levinson SR, Wynshaw-Boris A, Morris HR, Le D, Dell A, Schachter H, Marth JD (2001) Modeling human congenital disorder of glycosylation type IIa in the mouse: conservation of asparagine-linked glycan-dependent functions in mammalian physiology and insights into disease pathogenesis, Glycobiology 11: 1051-1070

28. Watt FM (1983) Involucrin and other markers of keratinocyte terminal differentiation. J Invest Dermatol 81: 100s-103s

29. Yamamoto S, Hase S, Fukuda S, Sano O, Ikenaka T (1989) Structures of the sugar chain of interferon- $\gamma$ produced by human myelomonocyte cell line HBL-38, J Biochem 105: 547-555 


\section{Figure Legends}

Figure 1. Chromatograms of $\mathrm{N}$-glycans from the epidermis.

Fluorescence tagged glycans released from the epidermis of patients and normal controls on an ODS column using HPLC. Peaks a, b and c are corresponding to $\mathrm{N}$-glycans, Gal $\beta 1,4$ GlcNAc $\beta 1,2$ Man $\alpha 1,3(G a l \beta 1,4 G l c N A c \beta 1,2 M a n \alpha 1,6) M a n \beta 1,4 G l c$ NAc $\beta 1,4$ GlcNAc,

Gal $\beta 1,4$ GlcNAc $\beta 1,2$ Man $\alpha 1,3($ Man $\alpha 1,6) M a n \beta 1,4 G l c N A c \beta 1,4(F u c \alpha 1,6) G$ lcNAc and

Gal $\beta 1$,4GlcNAc $\beta 1,2 M a n \alpha 1,3(G a l \beta 1,4 G l c N A c \beta 1,2 M a n \alpha 1,6) M a n \beta 1,4 G l c$ NAc $\beta 1,4($ Fuc $\alpha 1,6)$ GlcNAc, respectively.

Figure 2. Rates of molecular compartments, a, b and c.

Compartments $\mathrm{a}$ and $\mathrm{b}$ were increased and compartment $\mathrm{c}$ decreased in the hyperkeratotic diseased skin from patients. Colors of bar show following: slashed, a; white, b; black c. 

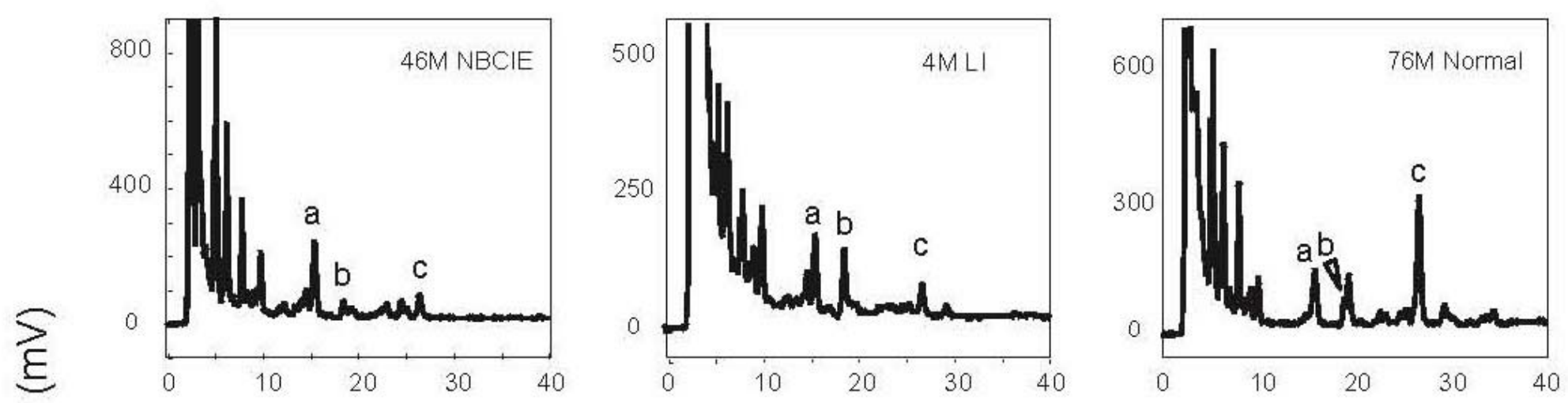

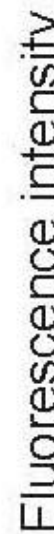
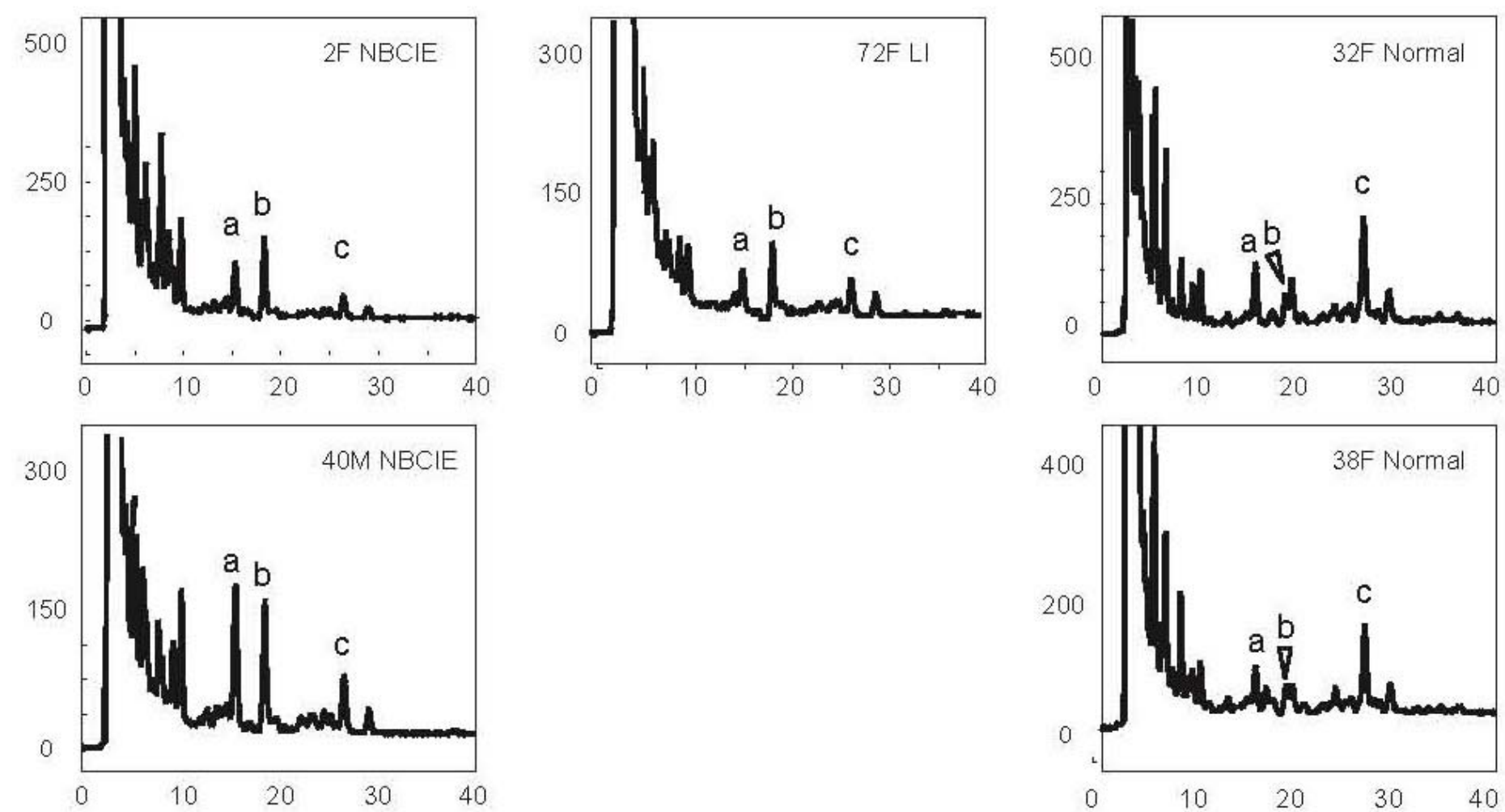

Elution time (min) 
